\title{
Radiosensitization of brain metastasis by targeting c-MET
}

\author{
Heekyoung Yang ${ }^{1,2,}{ }^{*}$, Hye Won Lee ${ }^{1,2,3,}{ }^{*}$, Yonghyun $\mathrm{Kim}^{4}$, Yeri Lee ${ }^{1,2,3}$, Yeon-Sook Choi ${ }^{1,2}$, Kang Ho Kim ${ }^{1,2}$, \\ Juyoun Jin ${ }^{1,2}$, Jeongwu Lee ${ }^{5}$ Kyeung Min Joo ${ }^{2,3,6,7}$ and Do-Hyun Nam ${ }^{1,2,3}$
}

Radiotherapy is the most widely used therapeutic modality in brain metastasis; however, it only provides palliation due to inevitable tumor recurrence. Resistance of tumor cells to ionizing radiation is a major cause of treatment failure. A critical unmet need in oncology is to develop rationale driven approaches that can enhance the efficacy of radiotherapy against metastatic tumor. Utilizing in vivo orthotopic primary tumor and brain metastasis models that recapitulate clinical situation of the patients with metastatic breast cancer, we investigated a molecular mechanism through which metastatic tumor cells acquire resistance to radiation. Recent studies have demonstrated that the hepatocyte growth factor (HGF)-c-Met pathway is essential for the pathologic development and progression of many human cancers such as proliferation, invasion and resistance to anticancer therapies. In this study, c-Met signaling activity as well as total c-Met expression was significantly upregulated in both breast cancer cell lines irradiated in vitro and ex vivo radio-resistant cells derived from breast cancer brain metastatic xenografts. To interrogate the role of c-Met signaling in radioresistance of brain metastasis, we evaluated the effects on tumor cell viability, clonogenicity, sensitivity to radiation, and in vitro/in vivo tumor growth after targeting c-Met by small-hairpin RNA (shRNA) or small-molecule kinase inhibitor (PF-2341066). Although c-Met silencing or radiation alone demonstrated a modest decrease in clonogenic growth of parental breast cancers and brain metastatic derivatives, combination of two modalities showed synergistic antitumor effects resulting in significant prolongation of overall survival in tumor-bearing mice. Taken together, optimizing c-Met targeting in combination with radiation is critical to enhance the effectiveness of radiotherapy in the treatments of brain metastasis.

Laboratory Investigation (2013) 93, 344-353; doi:10.1038/labinvest.2012.180; published online 4 February 2013

KEYWORDS: breast cancer brain metastasis; c-Met; radioresistance

Traditional estimates of the incidence of brain metastasis in patients with breast cancer range from 10 to $16 \%$ of metastatic patients. The incidence will be likely rising due to several factors including the improved neuroimaging facilities, an aging population, and more effective systemic treatment for the primary disease. ${ }^{1-5}$ Despite significant advances in understanding the molecular mechanisms of metastasis and development of new diagnostic, prognostic, and therapeutic tools, brain metastases constitute a major impact on morbidity and mortality, resulting in $\sim 7$ months median survival with significant cognitive impairment at the time of presentation. ${ }^{6-9}$

Up to now, standard treatment options for brain metastasis to aim locoregional control of cerebral metastases, palliation of symptoms, and prevention and/or improvement of functional deficits, include surgery, radiosurgery, and whole brain radiotherapy (WBRT) in various combinations. WBRT has been a critical component in the treatments of breast

\footnotetext{
${ }^{1}$ Department of Neurosurgery, Samsung Medical Center, Sungkyunkwan University School of Medicine, Seoul, South Korea; ${ }^{2}$ Cancer Stem Cell Research Center, Samsung Medical Center, Sungkyunkwan University School of Medicine, Seoul, South Korea; ${ }^{3}$ Samsung Medical Center, Samsung Advanced Institute for Health Sciences and Technology (SAIHST), Sungkyunkwan University School of Medicine, Seoul, South Korea; ${ }^{4}$ Department of Chemical and Biological Engineering, The University of Alabama, Tuscaloosa, AL, USA; ${ }^{5}$ Department of Stem Cell Biology and Regenerative Medicine, Lerner Research Institute, Cleaveland Clinic, Cleveland, OH, USA; ${ }^{6}$ Department of Anatomy, Sungkyunkwan University School of Medicine, Suwon, South Korea and ${ }^{7}$ Center for Molecular Medicine, Samsung Biomedical Research Institute, Suwon, South Korea

Correspondence: Professor KM Joo, Department of Anatomy, Sungkyunkwan University School of Medicine, 50 Irwon-dong, Seoul, Gangnam-gu 135-710, South Korea. E-mail: kmjoo@skku.edu or Professor D-H Nam, Department of Neurosurgery, Samsung Medical Center, Sungkyunkwan University School of Medicine, 50 Irwon-dong, Seoul, Gangnam-gu 135-710, South Korea.

E-mail: nsnam@skku.edu

"These authors contributed equally to this work.

Received 4 June 2012; revised 4 October 2012; accepted 26 October 2012
} 
cancer patients with all stages of the disease. Indeed, it is a gold standard modality in patients with multiple brain metastases for several decades since the recent meta-analysis results demonstrated the improved overall survival (OS) in these patients. ${ }^{10-13}$ However, the prolonged survival of the patients cannot be achieved always due to the recurrent or persistent tumor. Even when the tumor burden is decreased by radiation, many patients suffer cognitive and functional deficits caused by treatment-induced brain damage., ${ }^{74,15}$ Therefore, identifying radiation sensitizing agents to improve the efficacy of WBRT is an area of intense interest.

Cancer cells recognize DNA lesions induced by ionizing radiation (IR), and repair these lesions by activating various DNA repair pathways. ${ }^{16}$ Hyperactivation of these DNA repair pathways is a mechanism that confers radioresistance to cancer cells. A phenotypic switch of carcinoma cells to mesenchymal-like cells via epithelial-to-mesenchymal transition (EMT) is another mechanism associated with the acquisition of resistance. ${ }^{17}$ In addition, it was reported that the reduced production of reactive oxygen species (ROS), especially in a sub-population of highly tumorigenic, stemlike cells (called cancer initiating/stem cells), could protect the cancer cells from radiation-induced damage. ${ }^{18-23}$

c-Met, which is markedly overexpressed in aggressive forms of major human cancers, is a receptor tyrosine kinase with high affinity for hepatocyte growth factor (HGF) gaining particular attention because of its prominent role in invasion, metastasis and especially radioresistance. ${ }^{24} \mathrm{HGF} /$ c-Met signaling is known to be associated with EMT, and highly active in various stem cells. ${ }^{25}$ Recent study demonstrated that irradiation (IR) directly activates c-Met signaling by transcriptional upregulation of c-Met through the ATM-NF- $\kappa$ B signaling. ${ }^{26}$ The activated $\mathrm{c}-\mathrm{Met}$ signaling in turn triggers the activation of multiple c-Met downstream effectors including Ras/MAPK, AKT, and STAT3, which results in the promotion of cell survival and invasion, and the protection of tumor cells from DNA damage-induced apoptosis. ${ }^{26,27}$ Together, these studies suggest that targeted blockade of the HGF/c-Met pathway enhances therapeutic efficacy of radiotherapy by overcoming radioresistance.

Here, we elucidated the role of c-Met in radioresistance observed during the treatment of brain metastasis in the patients with breast cancer. In our study, c-Met expression and pathway activity in breast cancer cell lines and radioresistant cells derived from breast cancer brain metastatic xenografts were upregulated in response to IR, and the activated c-Met signaling protected tumor cells from radiationinduced apoptosis. Targeting c-Met by small-hairpin RNA (shRNA) or small-molecule kinase inhibitor (PF-2341066) combined with irradiation elicited synergistic antitumor response including inhibition of in vitro clonogenicity and effective tumor regression in both primary breast cancer and brain metastasis xenograft models, suggesting that c-Met silencing is a promising therapeutic option functioning as a radiosensitizer.

\section{MATERIALS AND METHODS}

\section{Cell Lines Culture and Isolation of Ex Vivo Cells}

Human metastatic cancer cell lines (a breast cancer cell line MDA-MB-231 and a putative breast/melanoma cell line MDA-MB-435) were purchased from ATCC. The parental cells, their subclonal cells expressing scrambled or c-Mettargeting shRNA, and ex vivo cells derived from xenograft tumors were grown in MEM or DMEM supplemented with $10 \%$ fetal bovine serum (FBS), $2 \mathrm{mM}$ L-glutamine, penicillin (100 U/ml), and streptomycin $(100 \mu \mathrm{g} / \mathrm{ml})$.

In in vivo radiation treatment experiments, mouse was killed when the body weight was decreased over 20\%. For obtaining tumor cells from the xenograft, we excised brain tissues and dissociated mechanically by chopping them with scissors. Dissociated sample was incubated with enzyme mixture solution with equal volume of sample for $30 \mathrm{~min}$. Enzyme mixture solution was $200 \mathrm{IU} / \mathrm{ml}$ collagenase, $0.1 \mathrm{IU} /$ $\mathrm{ml}$ DNaseI, $0.5 \mathrm{mg} / \mathrm{ml}$ Dispase and DPBS (2:0.1:0.5:47.4, v/v). After being washed with PBS, the minced tissue was filtered with a $0.2-\mu \mathrm{m}$ strainer and the resultant tumor cells were purified with Percoll (Sigma, Saint Louis, MO, USA). Ex vivo cells of MDA-MB-435 xenograft tumors were maintained with MEM containing $10 \%$ FBS, $100 \mathrm{U} / \mathrm{ml}$ penicillin, and $100 \mu \mathrm{g} / \mathrm{ml}$ streptomycin.

\section{In Vivo Xenograft Models}

To produce breast cancer orthotopic or brain metastatic animal models, 6-week-old female athymic nude mice were used. All experiments were conducted in accordance with the Institute for Laboratory Animal Research Guide for the Care and Use of Laboratory Animals and within the protocols approved by the appropriate Institutional Review Boards at the Samsung Medical Center (Seoul, Korea). For the orthotopic animal model, anesthetized mice were injected with subclones of expressing scramble or c-Met shRNA $\left(1 \times 10^{6} \%\right.$ $50 \mu \mathrm{l})$ into second thoracic mammary fat pad. When tumors reached about $250 \mathrm{~mm}^{3}$, animals were anesthetized and then irradiated with $10 \mathrm{~Gy}$ focused to tumor locally. Tumor diameter was measured using vernier calipers and tumor volume determined by calculating the volume of an ellipsoid using the formula: (length $\left.\times(\text { width })^{2} \times 0.5\right)$. For the brain metastatic animal model, subclones of expressing scramble or c-Met shRNA $\left(1 \times 10^{5} / 5 \mu \mathrm{l}\right)$ were stereotactically injected into the left striata of mice (coordinates; $\mathrm{AP}+1.0, \mathrm{ML}+1.7$, $\mathrm{DV}-3.2 \mathrm{~mm}$ from Bregma). At 15 days after cell injection, mice received $10 \mathrm{~Gy}$ of whole brain irradiation. Mice were killed either when $20 \%$ body weight loss or moribund status was observed. For analysis of tumor mass volume and apoptosis level in the brain metastatic model, three mice were killed at 5 days after whole brain irradiation. The brains of these mice were removed and processed for paraffin embedding. For analysis of tumor mass volume, standard $\mathrm{H} \& \mathrm{E}$ staining was performed in the paraffin. The DeadEnd fluorometric TUNEL system (Promega, Madison, WI, USA) 
was used to assay apoptosis and observed under optical microscope at $\times 400$ magnification.

\section{Depletion of c-Met Using shRNA}

The DNA sequence of c-Met-targeting shRNA (c-Met shRNA) is $5^{\prime}$-AGAATGTCATTCTACATGAGC- ${ }^{\prime}$. The retroviral vectors (pSuperRetro vector; Oligogene, Seattle, WA, USA) expressing the scrambled or c-Met shRNA have a pPGK backbone with an inserted H1 RNA promoter that drives shRNA expression. Cells transfected with scramble or c-Met shRNA were selected with $4 \mu \mathrm{g} / \mathrm{ml}$ puromycin (Invitrogen, Camarillo, CA, USA).

\section{Irradiation}

Human breast cancer cells, their subclones expressing scrambled or c-Met shRNA, or mice were irradiated using IBL 437C blood Irradiator (CIS US, Inc., Bedford, MA, USA) at a dose rate of $2.3 \mathrm{~Gy} / \mathrm{min}$.

\section{Clonogenic Assay}

To evaluate clonogenic potential of the irradiated cells, we used the assay described by Franken et al. ${ }^{28}$ Briefly, 50 cells were seeded in 6-well plates. After $18 \mathrm{~h}$, cells were irradiated (1-4Gy) in the presence of PF-2341066 $(500 \mathrm{nM})$ or the vehicle alone (DMSO), and then cultured for 14 days. Colonies containing $>50$ cells were counted as a representative of clonogenic cells. The survival fraction was calculated using the following formula: ((number of colonies formed after radiation)/(number of cells seeded $\times$ plating efficiency)), where plating efficiency is the ratio of seeded cells that gave rise to clones under no radiation conditions.

\section{qRT-PCR Analysis}

At $28 \mathrm{~h}$ after radiation, RNAs were extracted (Qiagen, Valencia, CA, USA) from radiated cells and their complementary DNAs were synthesized (Invitrogen) as per manufacturers' instructions. Quantitative reverse transcriptase PCR was performed using primers (sense; 5'TGGGAAGAAGATCACGAAG- $3^{\prime}$ antisense; $5^{\prime}$-TGTAGATTG CAGGCAGACAGA-3') in LightCycler 480 (Roche, Indianapolis, IN, USA).

\section{Flow Cytometry Analysis}

For detection of c-Met, cells were collected at $48 \mathrm{~h}$ after radiation (5 and $20 \mathrm{~Gy}$ ) and then fixed with $4 \%$ paraformaldehyde. Intensity of c-Met was detected with mouse anti-human c-Met polyclonal antibody (R\&D Systems, Minneapolis, MN, USA) conjugated with APC (eBiosciences, San Diego, CA, USA).

\section{Immunoblots}

To estimate upregulation or activation of c-Met in response to radiation, we analyzed protein expression and phosphorylation of c-Met in MDA-MB-231, MDA-MB-435, or ex vivo cells from breast cancer brain metastasis model. MDA-MB-
231 and MDA-MB- 435 cells were serum starved for $5 \mathrm{~h}$ and then irradiated with 5, 10 or $15 \mathrm{~Gy}$. Ex vivo cells and irradiated MDA-MB-231 and MDA-MB-435 cells were collected and lysed in RIPA Buffer (50 mM Tris-HCl, pH 7.5, $150 \mathrm{mM}$ $\mathrm{NaCl}, 2 \mathrm{mM}$ EDTA, $1 \%$ Triton X-100, 1\% sodium deoxycholate, $0.1 \%$ SDS) containing $50 \mathrm{mM} \mathrm{NaF}, 1 \mathrm{mM} \mathrm{Na}_{3} \mathrm{VO}_{4}$, $0.5 \%$ NP-40, and protease inhibitor cocktail tablets (Roche) after treatment of $100 \mathrm{ng} / \mathrm{ml} \mathrm{HGF}$ for $15 \mathrm{~min}$. Protein concentration was determined using a Bradford protein assay (Bio-Rad Laboratories, Hercules, CA, USA). Equal amounts of proteins were separated by SDS-PAGE and analyzed by immunoblotting with the following antibodies: rabbit polyclonal anti-Met (1:500; Abcam, San Francisco, CA), rabbit monoclonal anti-phospho-Met (Tyr1234/1235) (1:500; Cell Signaling Technology, Danvers, MA, USA), rabbit monoclonal anti-phospho-Met (Tyr1349) (1:500; Cell Signaling Technology). A mouse monoclonal anti-GAPDH (1:1000; Abcam) was used for the internal control. Antibodies were visualized with appropriate horseradish peroxidase-conjugated secondary antibodies (Pierce, Rockford, IL, USA), and the SuperSignal West Dura Chemiluminescent Substrate (Pierce).

\section{Statistics}

Numerical results were expressed as mean values \pm standard deviation (s.d.). Statistical comparisons were analyzed by one-way analysis of variance (ANOVA) followed by the least significant difference (LSD) test. Kaplan-Meier estimates of the survival function were plotted, and the significance of differences in OS was calculated by the Mantel-Cox log-rank test. A significance level of $P<0.05$ was used for all test. SPSS-PASW statistics software version 18.0 was used for all the statistical analyses.

\section{RESULTS}

\section{Radiation Upregulates c-Met Expression and Signaling Activity in Human Breast Cancer Cell Lines and Radio- Resistant Cells Derived from Breast Cancer Brain Metastatic Xenograft Model}

To investigate the role of c-Met in the response to IR, we first measured c-Met expression after in vitro irradiation (5 and 20 Gy) by flow cytometry analysis (Figures $1 \mathrm{a}$ and $\mathrm{b}$ ). In both MDA-MB-231 and MDA-MB-435 cells, the proportion of c-Met-positive cells ( 1.5 and 2.5 folds for 5 and $20 \mathrm{~Gy}$, respectively) and c-Met transcription level (1.8 and 2.6 folds, for 5 and $20 \mathrm{~Gy}$, respectively) increased dose dependently after irradiation (Figure 1c). The upregulation of c-Met and activation of c-Met signaling were confirmed in irradiated MDA-MB-231 and MDA-MB-435 cells by immunoblotting (Figure 1d).

To further investigate the role of c-Met in a more clinically relevant setting, we established the brain metastasis orthotopic models, in which in vivo irradiation was administered similar dose to WBRT in the human patients. Brain metastatic xenografts were generated by injection of MDA-MB- 
a

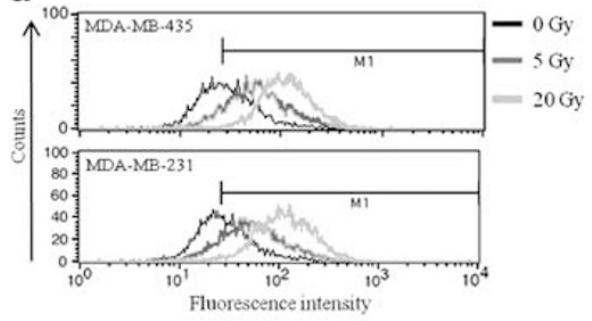

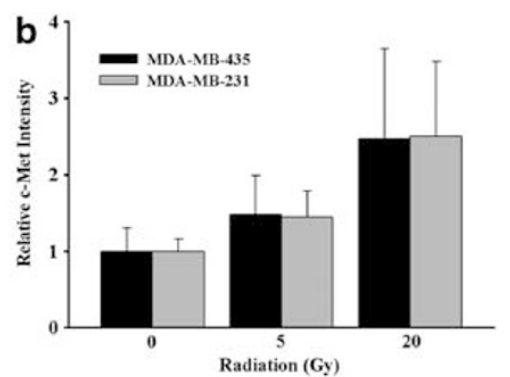

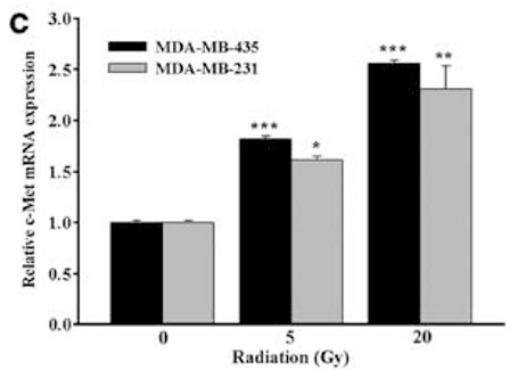

d

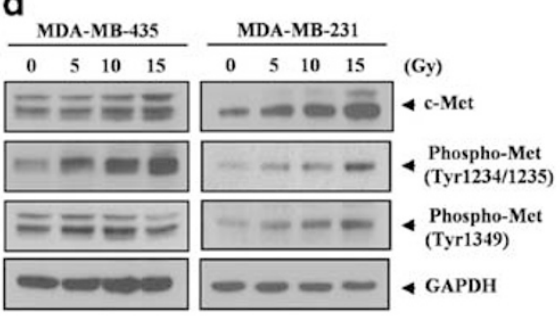

Figure 1 Increase of c-Met in respond to radiation in MDA-MB-435 and MDA-MB-231 breast cancer cells. (a) Representative histogram of c-Met fluorescence. (b) Intensity of c-Met was analyzed with flow cytometry. (c) mRNA levels of c-Met were analyzed qRT-PCR. (d) Expression and activation of $\mathrm{c}-$ Met in response to radiation were analyzed by immunoblotting. Values are mean \pm s.d., ${ }^{*} P<0.05,{ }^{* *} P<0.01,{ }^{* * *} P<0.001$ vs $0 \mathrm{~Gy}$.

a

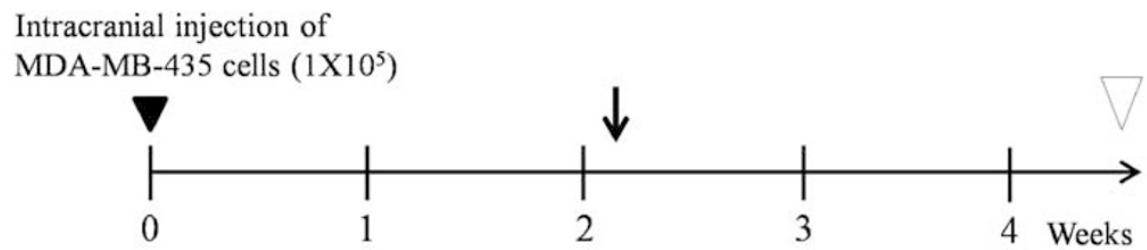

Whole brain radiation (10 Gy) at 15 day after tumor cell injection

Dissociation of tumor cell from individual mice at the day, which BW was lost over $20 \%$
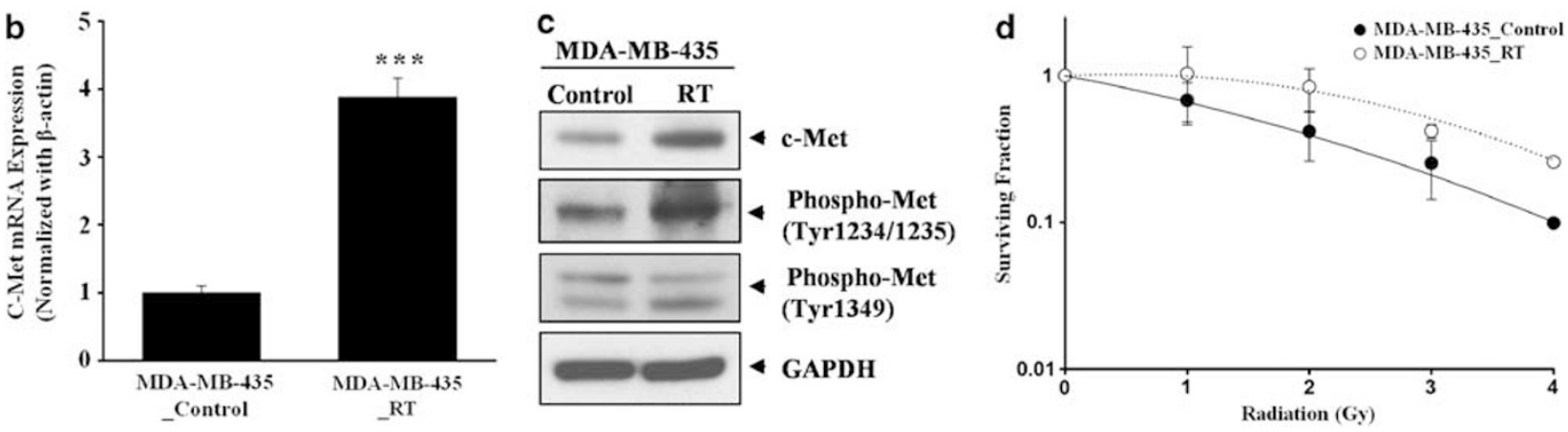

Figure 2 Upregulation of c-Met in radio-resistant ex vivo breast cancer cells. (a) Detailed experimental schedule for preparation of ex vivo cells. (b) mRNA levels of c-Met increased in radio-resistant ex vivo cells. (c) Expression and activation of c-Met in ex vivo cells were analyzed by immunoblotting. (d) Estimation of radioresponse of ex vivo cells by clonogenic survival assay. Values are mean \pm s.d., ${ }^{* * *} P<0.001$ vs Control.

435 cells via intracranial route and irradiation localized to whole brain (10 Gy) was performed 15 days after tumor inoculation (Figure 2a). We harvested residual xenograft tumors after radiation, dissociated them into single cells, and then characterized the isolated cells as radioresistant derivates established in vivo brain microenvironment (MDA435-RT). Similar with in vitro response to IR, these ex vivo cells demonstrated significant c-Met overexpression and enhanced signaling activity (Figures $2 \mathrm{~b}$ and $\mathrm{c}$ ). When in vitro clonogenic assays were performed with irradiation up to $4 \mathrm{~Gy}$, 

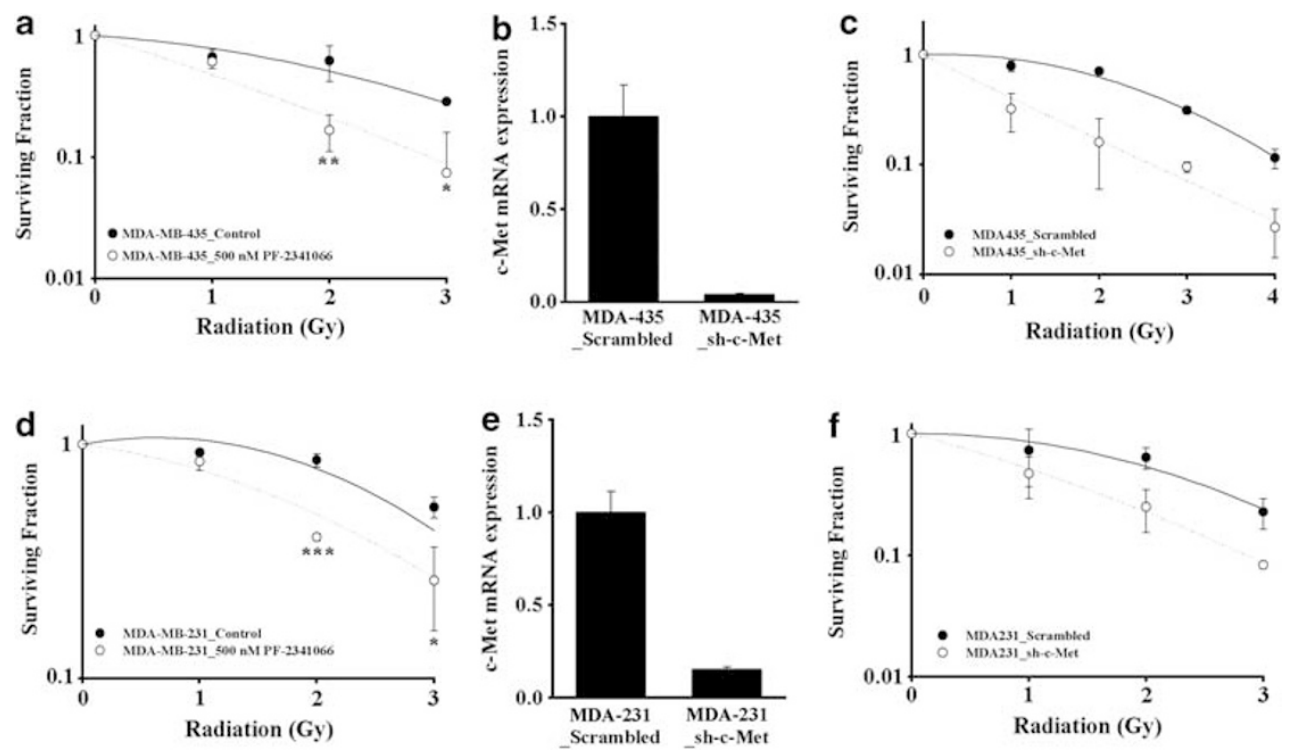

Figure 3 In vitro effects of c-Met inhibition in radiosensitivity of breast cancer cells. (a, d) PF-2341066, a c-Met tyrosine kinase inhibitor, enhanced radiosensitivity of MDA-MB-435 and MDA-MB-231 cells, respectively. (b, e) c-Met mRNA was decreased by c-Met shRNA expression in MDA-MB-435 and MDA-MB-231 cells, respectively. (c, f) Clonogenic survival assays were performed to estimate radioresponse in MDA-MB-435 and MDA-MB-231 cells, respectively. Values are mean \pm s.d., ${ }^{*} P<0.05,{ }^{* *} P<0.01,{ }^{* * *} P<0.001$ vs Control.

MDA435-RT cells generated much more colonies after irradiation than MDA435 control, suggesting that MDA435-RT cells acquired radio-resistant phenotypes (Figure 2d). Taken together, these findings indicate a potential causative role of c-Met in inducing resistance to radiation.

\section{Silencing of c-Met could Overcome Radioresistance in Breast Cancer Cells}

To further interrogate the role of c-Met signaling in radioresistance, the inhibition of clonogenic potential in response to IR was evaluated after targeting c-MET signaling by shRNAmediated knockdown or c-Met tyrosine kinase inhibitor, PF2341066. We have established c-Met-depleted cancer cell lines using shRNA (MDA435_sh-c-Met and MDA231_sh-c-Met) and then confirmed the efficiency of Met knockdown by quantitative RT-PCR (Figures $3 \mathrm{~b}$ and e). Data from the clonogenic assays combined with various doses of irradiation revealed that inhibition of c-Met activity by PF-2341066 (Figures $3 \mathrm{a}$ and $\mathrm{d}$ ) or RNA interference-mediated c-Met depletion (Figure $3 \mathrm{c}$ and $\mathrm{f}$ ) in both cell lines substantially reduced clonogenic fraction after same dose of irradiation as compared with their corresponding control cells, indicating that silencing of c-Met could sensitized tumor cells to IR. Furthermore, inhibition of c-Met in MDA435-IR cells significantly decreased the clonogenic survival after irradiation compared with its control (MDA435-IR_Scrambled) (data not shown).

\section{c-Met Inhibition Combined with Radiation Elicits a Synergistic Antitumor Response in Breast Cancer Orthotopic Xenograft Model}

A significant decrease in clonogenicity of cancer cells after combination of MET targeting and irradiation prompted us to test whether these antitumor responses can be achieved in vivo. Toward this goal, we first established xenograft tumors by injecting MDA435_Scrambled or MDA435_ sh-c-Met cells into mammary fat pad of nude mice (Figure 4a). Although the true identity of MDA435 is in debate, these cells behave as a highly malignant, metastatic tumor with shared characteristic of breast cancer cells. ${ }^{29,30}$ Once the tumors were established, we irradiated these tumors and evaluated the effects on tumor size. C-Met targeting alone did not produce a significant difference in tumor size (Figure 4c). Radiation affected the tumor growth, as expected. Remarkably, tumors treated with both irradiation and c-Met knockdown were significantly smaller than those of any other groups (53\% smaller than MDA435_Scrambled_no RT; 43\% smaller than MDA435_sh-c-Met_no RT; 38\% smaller than MDA435_Scrambled_10 Gy), indicating a potent radiosensitization by c-Met targeting (Figures $4 \mathrm{~b}$ and $\mathrm{c}$ ).

\section{c-Met Inhibition Combined with Radiation Elicits a Synergistic Antitumor Response in Brain Metastatic Tumor Model by Increasing Radiation-Induced Tumor Cell Apoptosis}

Next, we analyzed the radiosensitizing effect of c-Met depletion in brain metastasis orthotopic model (Figure $5 \mathrm{a}$ ). The survival of tumor-bearing mice was monitored after MDAMB-435 cells with or without c-Met silencing was directly implanted to the brains of mice. As shown in Figure 5b, similarly to typical clinical response in human patients with brain metastasis, irradiation alone did not confer significant survival gains (median survival of 26 vs 23). Mice injected with MDA435_sh-c-Met cells combined with in vivo irradiation (median survival of 37) survived significantly longer 


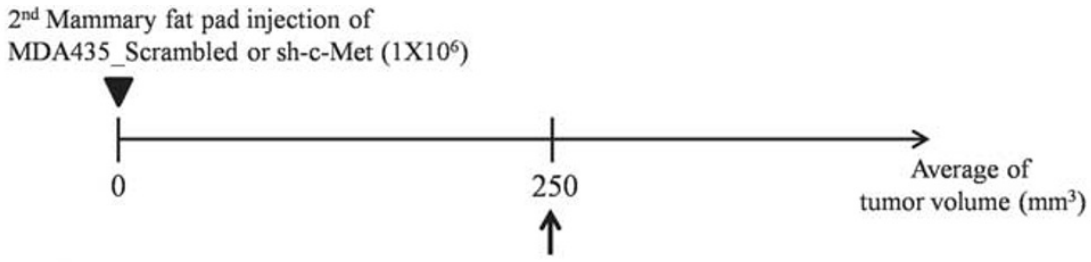

$\uparrow$ Grouping and radiation (10 Gy) at the day, which tumor reaches $250 \mathrm{~mm}^{3}$

b

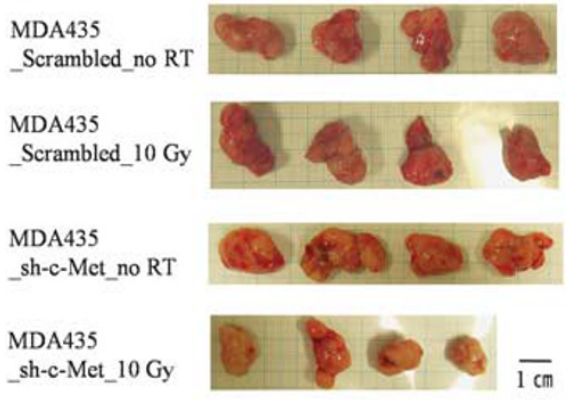

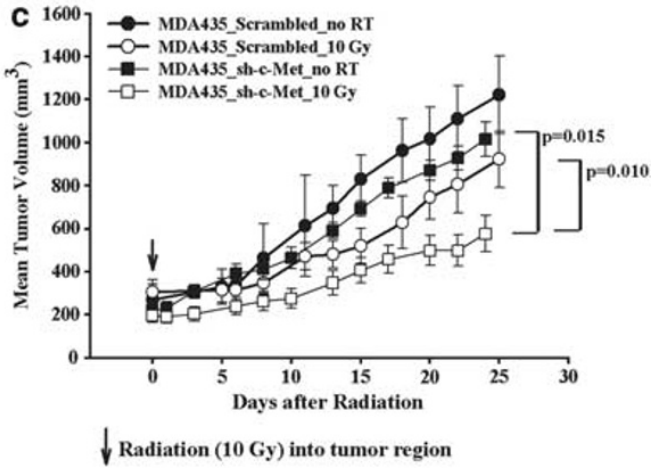

Figure 4 In vivo effects of c-Met inhibition on radiosensitivity of breast cancer orthotopic xenografts. (a) Detailed experimental schedules.

(b) Representative images of tumor mass. (c) Tumor growth after radiation in MDA-MB-435 breast cancer orthotopic models. Values are mean \pm s.e.

a Intracranial injection of

MDA435_Scrambled or sh-c-Met $\left(1 \times 10^{5}\right)$

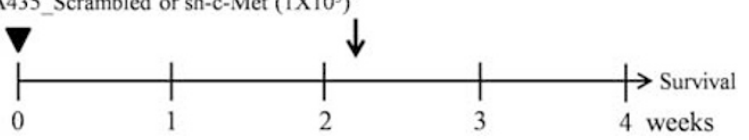

$\downarrow$ Whole brain radiation (10 Gy) at 15 day after tumor cell injection

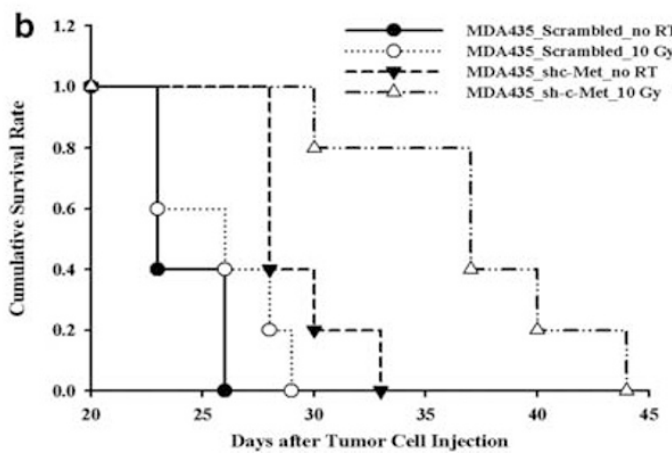

Figure 5 Inhibition of c-Met enhanced radiosensitivity in breast cancer brain metastatic model. (a) Detailed experimental schedules. (b) KaplanMeier plot comparing survival of MDA-MB-435 brain metastatic xenograft models.

than mice of any other groups $(P<0.01$ vs MDA435 Scrambled_no RT; $P<0.01$ vs MDA435_Scrambled_10 Gy). We also measured tumor volumes of each group 5 days after whole brain irradiation (Figure 6a). A significant antitumor effect was shown in the mice treated with combined c-Met silencing and IR and complete tumor regression was observed in a few mice. The tumor volume in the combination group was significantly smaller while radiation or c-Met silencing alone resulted in the slightly reduced tumor volume, indicating a strong synergistic effect $(P<0.01$ vs MDA435 Scrambled + RT) (Figures $6 \mathrm{~b}$ and $\mathrm{c}$ ).

To investigate a potential reason of a strong antitumor effect shown above, we performed immunohistochemical analysis on the sections derived from the each group (Figures 5 and 6). Notably, determination of apoptotic cells by TUNEL assays revealed an interesting clue. As expected, radiation treatment increased the number of TUNEL-positive apoptotic cells compared with its non-irradiated control group $(P<0.01)$. In contrast, MDA435-c-Met_RT tumors revealed the significant increase in the number of TUNELpositive cells compared with MDA435_Scrambled + RT $(P<0.01)$ (Figure 7b). These data demonstrate that synergistic inhibition of tumor growth by a combination of c-Met silencing and radiation may be mediated in part by the increased incidence of tumor cell apoptosis.

\section{DISCUSSION}

Cancer metastasis is the single most important factor influencing cancer patient mortality. Controlling the metastatic spread of tumors is indeed one of the most important crucial aspects for the successful cancer treatment. In the patients with brain metastasis, attempts to control the size of tumor by irradiation, which might prolong OS, must be balanced with the consideration of quality of life and neurological function. With improvements in control of systemic disease of breast cancer and in survival, the brain has quickly emerging as a prime sanctuary site of tumor relapse in patients with otherwise controlled breast cancer. Although considerable progress has been made in the treatment of brain metastases, conservative management, chemotherapy 


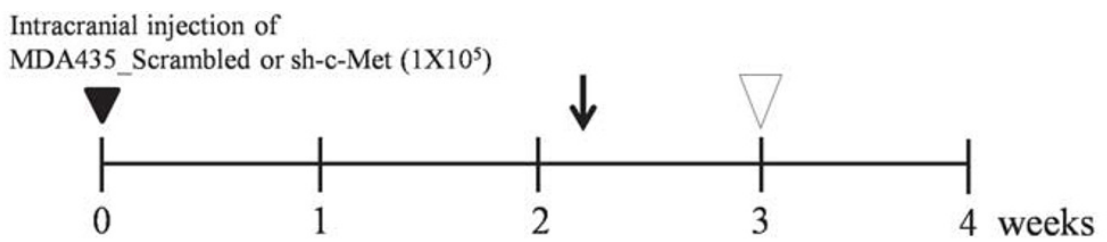

Whole brain radiation (10 Gy) at 15 day after tumor cell injection

Sacrifice of all mice and brain preparation from individual mice

b

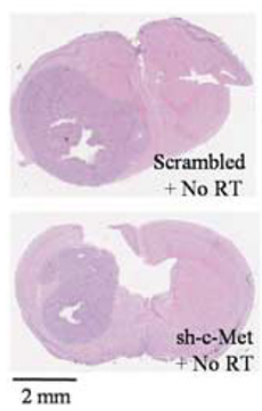

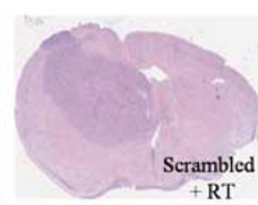

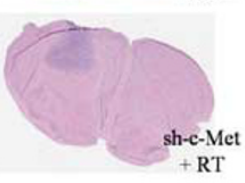

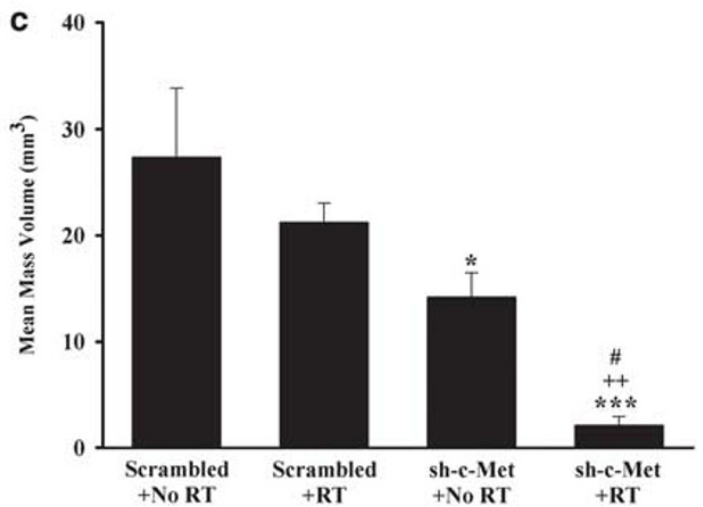

Figure 6 Tumor regression induced by combination of c-Met depletion and radiation in breast cancer brain metastatic xenograft model. (a) Detailed experimental schedule. (b) Representative images of brain tumors. The brain was stained with H\&E. (c) Tumor volumes of each group were analyzed. Values are mean \pm s.e. ${ }^{*} P<0.05,{ }^{* * *} P<0.001$ vs Scrambled + No $\mathrm{RT} ;{ }^{++} P<0.01$ vs Scrambled $+\mathrm{RT} ;{ }^{*} P<0.05$ vs sh-c-Met + No RT.
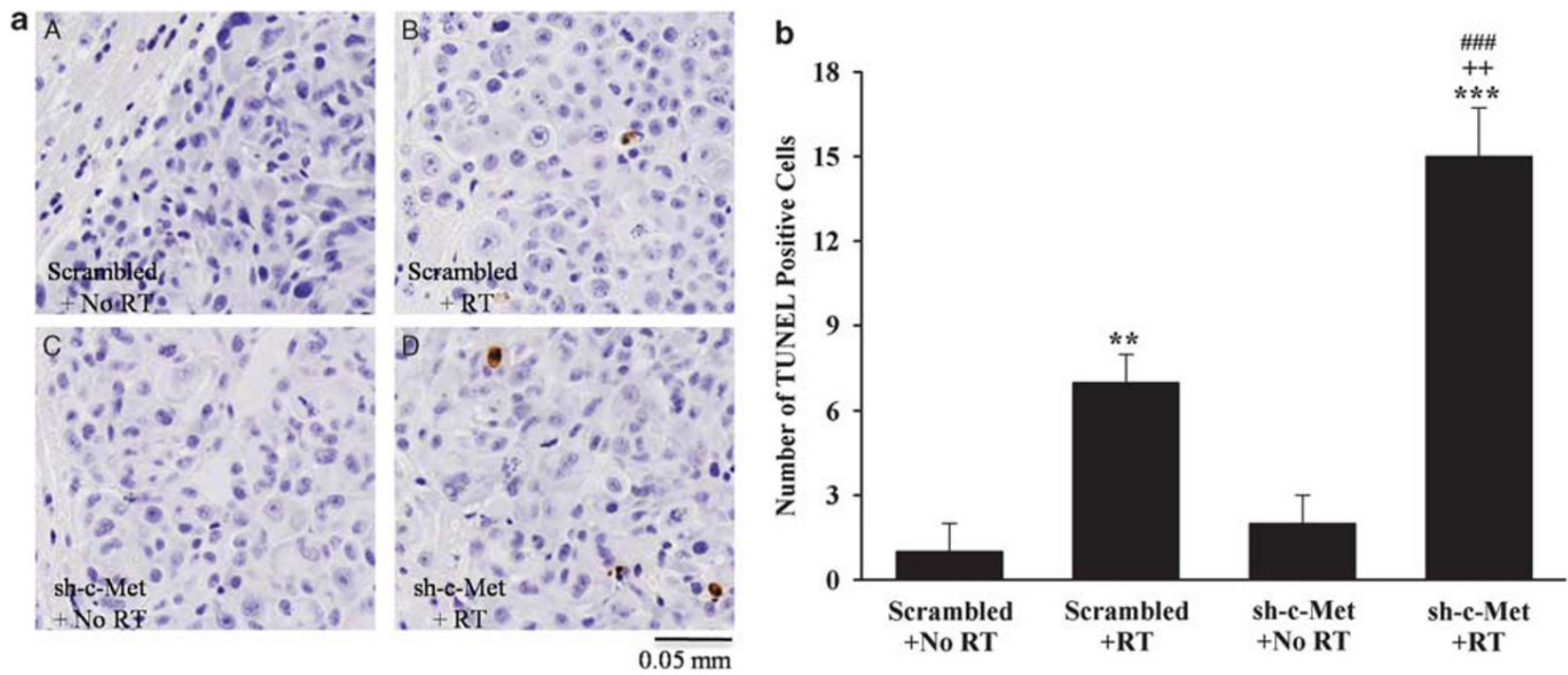

Figure 7 Induction of apoptosis by combination of c-Met depletion and radiation in breast cancer brain metastatic xenograft model. Apoptosis was detected by TUNEL assay. (a) Representative images for TUNEL-positive cells. (b) Number of TUNEL-positive cells in each group was analyzed. Values are mean \pm s.e. ${ }^{* *} P<0.01,{ }^{* * *} P<0.001$ vs Scrambled + No RT; ${ }^{++} P<0.01$ vs Scrambled $+\mathrm{RT} ;{ }^{\# \# \#} P<0.001$ vs sh-c-Met + No RT.

or radiotherapy remains the cornerstone of management in the majority of patients. Unfortunately, conventional cytotoxic anticancer therapies have shown an unsatisfactory outcome.

Radiotherapy provides an organ sparing approach and improved OS via an efficient local control of early-stage cancer, and it is also an effective means of palliating symp- toms of metastatic disease. ${ }^{31}$ Typically, about $80-90 \%$ of patients with metastatic breast experience significant clinical response to local radiotherapy, indicating that metastatic breast cancer is a radiosensitive disease. ${ }^{32}$ Brain metastasis is recently designated as an unmet medical need by the US Food and Drug Administration. The mainstay of treatment is radiation therapy; however, this treatment sometimes adds 
additional morbidity through neurocognitive complications caused by focal radiation necrosis and diffuse leukoencephalopathy from WBRT.7,14,15,33 The aim of radiation therapy is to maximize radiation dose to the tumor to achieve local control and minimize the dose to normal tissues in order to decrease treatment-related acute and late morbidity. ${ }^{34,35}$ For the treatment of brain metastases in patients with breast cancer, the development of simultaneously administered agents that can be used to enhance the effect of radiotherapy (eliciting either additive or synergistic effect) is urgently required. In recent decades, numerous clinical advances have been made due to the increased understanding of molecular regulation of radiation effects and the improved precision of dose delivery to the target tumor. IR has been shown to activate multiple signaling pathways, including MAPK, JNK, and PI3K, which in turn affect cell survival and mitogenic responses. ${ }^{36}$ The mechanisms causing tumor radio-adaptive resistance are attracting a great deal of interest because of its essential role in the efficacy of clinical anticancer radiotherapy.

Recent studies reported that concomitant use of cetuximab, an epidermal growth factor receptor (EGFR) inhibitor or an anti-EGFR monoclonal antibody, and radiotherapy significantly improved the treatment results of patients with head and neck carcinomas. ${ }^{37,38}$ As another effective radiosensitizing targeting agent, our study suggests that radiationinduced upregulation of c-Met and activation of c-Met signaling is associated with radioresistance of breast cancer cells, and that targeting HGF/c-Met pathway could be a potential radio-sensitizing therapeutic approach that generates synergistic antitumor activity in both primary mass and brain metastasis of breast cancer. Up to date, c-Met has been suggested as an independent prognostic factor in breast cancer. ${ }^{39,40}$ Although the detailed mechanism of induced c-Met upregulation by irradiation was not elucidated in this study, a recent report showed that c-Met expression is increased via activation of ATM and NF- $\kappa$ B. ${ }^{26}$ In another study, MET inhibition by a small-molecule Met inhibitor, PHA665752, reduced clonogenic survival of tumor cells when combined with IR via the attenuated post-damage DNA repair pathways. ${ }^{41}$ Increased apoptosis in tumor tissues after concomitant c-Met targeting and IR in our study suggests that c-Met silencing may impair the repair of DNA lesions including single-strand breaks (SSBs), double-strand breaks (DSBs), DNA base alterations, and DNA-DNA or DNAprotein cross-links produced by radiation.

Although amplification or overexpression of c-Met and/or HGF mediating paracrine or autocrine c-Met pathway activation is observed in almost all types of solid tumors, c-Met amplification or mutation causing constitutive activation also has been found in a few cancers. ${ }^{42}$ Various c-Met inhibiting drugs including neutralizing antibodies against either HGF or c-Met, and small-molecule inhibitors that antagonize c-Met kinase activity has been developed. ${ }^{43-45}$ The c-Met receptor is expressed by a wide variety of epithelial cells, whereas its ligand HGF is normally produced by the stromal tissues, and the interactions between cancer cells and surrounding tumor microenvironment involving the HGF/cMet pathway have been suggested to have a critical role in tumor aggressiveness. ${ }^{46}$ However, mouse HGF binds human c-Met with only low affinity and does not potently activate human MET signaling. ${ }^{47}$ In this study, we used a brain metastasis model established by intracranial injection of nonbrain origin tumors and tested the therapeutic efficacy of c-Met silencing combined with in vivo irradiation. The enhanced antitumor radiation responses seen in both primary tumor orthotopic and brain metastasis model that have the distinct tumor microenvironment (that is, mammary fat fad $v$ brain), may indicate that the radio-sensitizing response of $\mathrm{HGF} / \mathrm{c}-\mathrm{Met}$ pathway inhibition can be expected in both primary and metastatic tumors and that these effects would be mediated by inhibition of autocrine c-Met pathway activation or constitutive c-Met activation by amplification or mutation other than paracrine c-Met signaling activation.

In future studies, targetable signaling pathways including c-Met involved in tumor cell-stromal interactions in the brain and respective landscaping role in promoting brain metastasis should be investigated in detail. A transgenic mouse model expressing human HGF on an immunecompromised severe combined immunodeficient background has been developed as a preclinical in vivo test system for assessing efficacy c-Met drugs against various human cancers. ${ }^{48}$ In addition, novel administration method need to be developed that can enhance the delivery of the small molecular inhibitor into the brain mass bypassing the blood-brain barrier, as shRNA-mediated Met targeting is less likely to be translated in the clinic.

As c-Met is known to be a critical signaling molecule in normal stem cell function, the potential role of c-Met as a functional marker for cancer stem cell has been studied. We and others have shown that c-Met is a cancer stem cell marker in the pancreatic and brain cancers. ${ }^{49-51}$ Therapeutic efficacy of Met targeting in vivo was not evaluated in these studies. ${ }^{50,51}$ The potent antitumor activity elicited by c-Met silencing and irradiation shown in our study might be partly derived from targeting effect on putative cancer stem cells present in tumor in situ. In this aspect, the established cancer cell lines used in this study have limitation in terms of representing tumor heterogeneity and hierarchy. ${ }^{52,53}$ Until now, many researchers and pharmaceutical companies have been developing therapeutic drugs by using traditional and artificial model systems such as established cell lines and genetically modified cells. Our ongoing studies utilize the patient-derived breast cancer cells to build a more clinically relevant preclinical platform. Notwithstanding this caveat, our study provides preclinical evidence supporting the c-Met targeting combined with irradiation is a promising therapeutic approach against breast cancer and its metastasis to the brain. 


\section{ACKNOWLEDGEMENTS}

This work was supported by a grant from The Korea Healthcare Technology R\&D Project, Ministry for Health \& Welfare Affairs, Republic of Korea (A092255). This work was supported by the National Research Foundation of KOREA (NRF) grant funded by the Korea government (MEST)

(No. 20090093731)

\section{DISCLOSURE/CONFLICT OF INTEREST}

The authors declare no conflict of interest.

1. Arslan C, Dizdar O, Altundag K. Systemic treatment in breast-cancer patients with brain metastasis. Expert Opin Pharmacother 2010;11: 1089-1100.

2. Altundag $\mathrm{K}$, Bondy $\mathrm{ML}$, Mirza $\mathrm{NQ}$, et al. Clinicopathologic characteristics and prognostic factors in 420 metastatic breast cancer patients with central nervous system metastasis. Cancer 2007;110: 2640-2647.

3. Lagerwaard FJ, Levendag PC, Nowak PJ, et al. Identification of prognostic factors in patients with brain metastases: a review of 1292 patients. Int J Radiat Oncol Biol Phys 1999;43:795-803.

4. Shaffrey ME, Mut M, Asher AL, et al. Brain metastases. Curr Probl Surg 2004;41:665-741.

5. Chang EL, Wefel JS, Maor MH, et al. A pilot study of neurocognitive function in patients with one to three new brain metastases initially treated with stereotactic radiosurgery alone. Neurosurgery 2007;60: 277-283, discussion 283-274.

6. Palmieri D, Fitzgerald D, Shreeve SM, et al. Analyses of resected human brain metastases of breast cancer reveal the association between upregulation of hexokinase 2 and poor prognosis. Mol Cancer Res 2009;7:1438-1445.

7. Chang EL, Wefel JS, Hess KR, et al. Neurocognition in patients with brain metastases treated with radiosurgery or radiosurgery plus whole-brain irradiation: a randomised controlled trial. Lancet Oncol 2009:10:1037-1044.

8. Sperduto PW, Chao ST, Sneed PK, et al. Diagnosis-specific prognostic factors, indexes, and treatment outcomes for patients with newly diagnosed brain metastases: a multi-institutional analysis of 4,259 patients. Int J Radiat Oncol Biol Phys 2010;77:655-661.

9. Acosta-Jamett G, Astorga-Arancibia F, Cunningham AA. Comparison of chemical immobilization methods in wild foxes (Pseudalopex griseus and Pseudalopex culpaeus) in Chile. J Wildl Dis 2010;46: 1204-1213.

10. Debeb BG, Xu W, Woodward WA. Radiation resistance of breast cancer stem cells: understanding the clinical framework. J Mammary Gland Biol Neoplasia 2009;14:11-17.

11. Glynne-Jones R, Sebag-Montefiore D. Chemoradiation scheduleswhat radiotherapy? Eur J Cancer 2002;38:258-269.

12. Clarke M, Collins R, Darby $\mathrm{S}$, et al. Effects of radiotherapy and of differences in the extent of surgery for early breast cancer on local recurrence and 15-year survival: an overview of the randomised trials. Lancet 2005;366:2087-2106.

13. Gebski V, Lagleva M, Keech A, et al. Survival effects of postmastectomy adjuvant radiation therapy using biologically equivalent doses: a clinical perspective. J Natl Cancer Inst 2006;98:26-38.

14. Jenkinson MD, Haylock B, Shenoy A, et al. Management of cerebral metastasis: evidence-based approach for surgery, stereotactic radiosurgery and radiotherapy. Eur J Cancer 2011;47:649-655.

15. Ricard D, Taillia H, Renard JL. Brain damage from anticancer treatments in adults. Curr Opin Oncol 2009;21:559-565.

16. Zhu Y, Hu J, Hu Y, et al. Targeting DNA repair pathways: a novel approach to reduce cancer therapeutic resistance. Cancer Treat Rev 2009; 35:590-596.

17. Palena C, Fernando Rl, Litzinger MT, et al. Strategies to target molecules that control the acquisition of a mesenchymal-like phenotype by carcinoma cells. Exp Biol Med (Maywood) 2011;236: 537-545.

18. Bao $\mathrm{S}, \mathrm{Wu} \mathrm{Q}, \mathrm{McLendon} \mathrm{RE}$, et al. Glioma stem cells promote radioresistance by preferential activation of the DNA damage response. Nature 2006;444:756-760.
19. Phillips TM, McBride WH, Pajonk F. The response of CD24(-/low)/ CD44 + breast cancer-initiating cells to radiation. J Natl Cancer Inst 2006;98:1777-1785.

20. Creighton CJ, Li X, Landis $M$, et al. Residual breast cancers after conventional therapy display mesenchymal as well as tumor-initiating features. Proc Natl Acad Sci USA 2009;106:13820-13825.

21. Woodward WA, Chen MS, Behbod F, et al. WNT/beta-catenin mediates radiation resistance of mouse mammary progenitor cells. Proc Natl Acad Sci USA 2007;104:618-623.

22. Visvader JE, Lindeman GJ. Cancer stem cells in solid tumours: accumulating evidence and unresolved questions. Nat Rev Cancer 2008;8:755-768.

23. Al-Hajj M, Wicha MS, Benito-Hernandez A, et al. Prospective identification of tumorigenic breast cancer cells. Proc Natl Acad Sci USA 2003;100:3983-3988.

24. Vande Woude GF, Jeffers M, Cortner J, et al. Met-HGF/SF: tumorigenesis, invasion and metastasis. Ciba Found Symp 1997;212:119-130, discussion 130-112, 148-154.

25. Joo KM, Jin J, Kim E, et al. MET Signaling Regulates Glioblastoma Stem Cells. Cancer Res 2012;72:3828-3838.

26. De Bacco F, Luraghi P, Medico E, et al. Induction of MET by ionizing radiation and its role in radioresistance and invasive growth of cancer. J Natl Cancer Inst 2011;103:645-661.

27. Qian LW, Mizumoto K, Inadome N, et al. Radiation stimulates HGF receptor/c-Met expression that leads to amplifying cellular response to HGF stimulation via upregulated receptor tyrosine phosphorylation and MAP kinase activity in pancreatic cancer cells. Int J Cancer 2003;104:542-549.

28. Franken NA, Rodermond HM, Stap J, et al. Clonogenic assay of cells in vitro. Nat Protoc 2006;1:2315-2319.

29. Price JE, Polyzos A, Zhang RD, et al. Tumorigenicity and metastasis of human breast carcinoma cell lines in nude mice. Cancer Res 1990;50:717-721.

30. Price JE, Zhang RD. Studies of human breast cancer metastasis using nude mice. Cancer Metastasis Rev 1990;8:285-297.

31. Hartsell WF, Scott CB, Bruner DW, et al. Randomized trial of shortversus long-course radiotherapy for palliation of painful bone metastases. J Natl Cancer Inst 2005;97:798-804.

32. Rades $D$, Huttenlocher $S$, Dunst J, et al. Matched pair analysis comparing surgery followed by radiotherapy and radiotherapy alone for metastatic spinal cord compression. J Clin Oncol 2010;28:3597-3604.

33. Gril B, Evans L, Palmieri D, et al. Translational research in brain metastasis is identifying molecular pathways that may lead to the development of new therapeutic strategies. Eur J Cancer 2010;46:1204-1210.

34. Helleday T, Petermann E, Lundin C, et al. DNA repair pathways as targets for cancer therapy. Nat Rev Cancer 2008;8:193-204.

35. Liu SK, Olive PL, Bristow RG. Biomarkers for DNA DSB inhibitors and radiotherapy clinical trials. Cancer Metastasis Rev 2008;27:445-458.

36. Ahmed KM, Li JJ. NF-kappa B-mediated adaptive resistance to ionizing radiation. Free Radic Biol Med 2008:44:1-13.

37. Szumiel I. Epidermal growth factor receptor and DNA double strand break repair: the cell's self-defence. Cell Signal 2006;18:1537-1548.

38. Dittmann $\mathrm{K}$, Mayer $\mathrm{C}$, Fehrenbacher B, et al. Radiation-induced epidermal growth factor receptor nuclear import is linked to activation of DNAdependent protein kinase. J Biol Chem 2005;280:31182-31189.

39. Lengyel $E$, Prechtel $D$, Resau JH, et al. C-Met overexpression in nodepositive breast cancer identifies patients with poor clinical outcome independent of Her2/neu. Int J Cancer 2005;113:678-682.

40. Ghoussoub RA, Dillon DA, D'Aquila $T$, et al. Expression of c-met is a strong independent prognostic factor in breast carcinoma. Cancer 1998:82:1513-1520.

41. Medova Michaela, Aebersold DM, Blank-Liss Wieslawa, et al. MET inhibition results in DNA breaks and synergistically sensitizes tumor cells to DNA-damaging agents potentially by breaching a damageinduced checkpoint arrest. Genes Cancer 2011;1:1053-1062.

42. Birchmeier C, Birchmeier W, Gherardi E, et al. Met, metastasis, motility and more. Nat Rev Mol Cell Biol 2003;4:915-925.

43. Comoglio PM, Giordano S, Trusolino L. Drug development of MET inhibitors: targeting oncogene addiction and expedience. Nat Rev Drug Discov 2008;7:504-516.

44. Eder JP, Vande Woude GF, Boerner SA, et al. Novel therapeutic inhibitors of the c-Met signaling pathway in cancer. Clin Cancer Res 2009;15:2207-2214. 
45. Knudsen BS, Vande Woude G. Showering c-MET-dependent cancers with drugs. Curr Opin Genet Dev 2008;18:87-96.

46. Nakamura T, Matsumoto K, Kiritoshi A, et al. Induction of hepatocyte growth factor in fibroblasts by tumor-derived factors affects invasive growth of tumor cells: in vitro analysis of tumor-stromal interactions. Cancer Res 1997;57:3305-3313.

47. Rong S, Bodescot M, Blair D, et al. Tumorigenicity of the met protooncogene and the gene for hepatocyte growth factor. Mol Cell Biol 1992;12:5152-5158.

48. Zhang YW, Staal B, Essenburg C, et al. MET kinase inhibitor SGX523 synergizes with epidermal growth factor receptor inhibitor erlotinib in a hepatocyte growth factor-dependent fashion to suppress carcinoma growth. Cancer Res 2010;70:6880-6890.
49. Joo KyeungMin, Jin J, Kim Eunhee, et al. MET signaling regulates glioblastoma stem cells. Cancer Res 2012;72:3828-3838.

50. Suzuki A, Nakauchi $\mathrm{H}$, Taniguchi $\mathrm{H}$. Prospective isolation of multipotent pancreatic progenitors using flow-cytometric cell sorting. Diabetes 2004;53:2143-2152.

51. Boccaccio C, Comoglio PM. Invasive growth: a MET-driven genetic programme for cancer and stem cells. Nat Rev Cancer 2006;6:637-645.

52. Cree IA, Glaysher S, Harvey AL. Efficacy of anti-cancer agents in cell lines versus human primary tumour tissue. Curr Opin Pharmacol 2010:10:375-379.

53. Gillet JP, Calcagno AM, Varma $\mathrm{S}$, et al. Redefining the relevance of established cancer cell lines to the study of mechanisms of clinical anticancer drug resistance. Proc Natl Acad Sci USA 2011;108:18708-18713. 